Jpn Circ J 1997; 61: 78-81

\title{
Right Bundle Branch Block in A Patient With Acute Posterior Myocardial Infarction
}

\author{
Masanori Okabe, MD; Junko Nomoto. MD; Keisuke Fukuda, MD; \\ Kikuo Arakawa, MD; Masahiro Kikuchi, MD*
}

\begin{abstract}
We present here a patient with the unusual combination of a newly developed right bundle branch block (RBBB) and an acute posterior myocardial infarction (MI). A dissecting MI with interventricular septal (IVS) rupture was initially thought to have caused RBBB. However, histologic examination revealed that the right bundle branch (RBB) had no ischemic involvement, but did show fibrosis, which suggests that this chronic damage of the RBB was a major contributor to the development of RBBB. This case confirms that the RBB is rarely involved by a posterior MI even in the presence of a dissecting IVS rupture.
\end{abstract}

(Jpn Circ J 1997; 61: 78-81)

Key Words: Interventricular septal rupture; Right bundle branch block; Acute posterior myocardial Infarction

$\mathbf{W}^{\mathrm{b}}$ hile ischemic damage to the right bundle branch (RBB) is rarely associated with a posterior myocardial infarction (MI), it is sometimes associated with an anterior MI 1,2 This can be explained by the arterial blood supply to the interventricular septum (IVS) which is derived from the anterior and posterior descending arteries 3,4 The anterior descending artery feeds the anterior two-thirds of the IVS including the RBB, the anterior radiation of the left bundle branch (LBB), and part of the bundle of His. The posterior descending artery perfuses the posterior third of the IVS, including the atrioventricular (AV) node, the His bundle and the posterior radiation of the LBB. We present here a patient who developed right bundle branch block (RBBB) during the acute phase of an posterior MI.

\section{Case Report}

An 81-year-old Japanese man was admitted to a local hospital with a heart attack. Conservative treatment for acute MI was administrated. The patient's initial clinical course was uneventful: and the typical electrocardiographic evolution of an acute posterior MI was observed (Fig 1). However, on the sixth hospital day, he developed cardiogenic shock with a new systolic heart murmur. The electrocardiogram
(ECG) at this time showed atrial fibrillation, a reelevation of the ST segment in the inferior leads and reciprocal ST depression in the precordial leads. The patient was then transferred to Fukuoka University Hospital to receive intensive care. RBBB was present on admission. Use of a balloon-tipped catheter revealed a left-to-right shunt ratio of $60 \%$ at the ventricular level, which suggested a diagnosis of a ruptured IVS. Eight hours later, sinus rhythm was restored with a slight prolongation of the $\mathrm{PQ}$ interval $(0.24 \mathrm{sec})$. The presence of severe atherosclerosis of the abdominal aorta prevented the use of intra-aortic balloon counterpulsation. The patient's hemodynamic state had deteriorated and could not be stabilized long enough to permit surgical intervention. RBBB persisted for $23 \mathrm{~h}$ and the patient died.

Necropsy examination (Fig 2) confirmed the presence of MI of recent onset, and a mural hemorrhage was found in the posterior walls of the left and right ventricles. The epicardial coronary arteries showed 3vessel disease with recent thrombosis in the proximal portion of the dominant right coronary artery. A small perforation was found in the IVS on the right ventricular side. Marked myocardial dissection was observed toward the posterior edge of the uppermost IVS on the left ventricular side. The MI extended anteriorly far enough to reach the posterior part of the membranous

(Received February 5, 1996; accepted May 29, 1996)

Departments of Internal Medicine and "Pathology, School of Medicine, Fukuoka University Fukuoka, Japan

Mailing address: Masanori Okabe, M.D., Department of Internal Medicine School of Medicine, Fukuoka University 7-45-1, Nanakuma, Jonan-ku Fukuoka, 814-01, Japan 


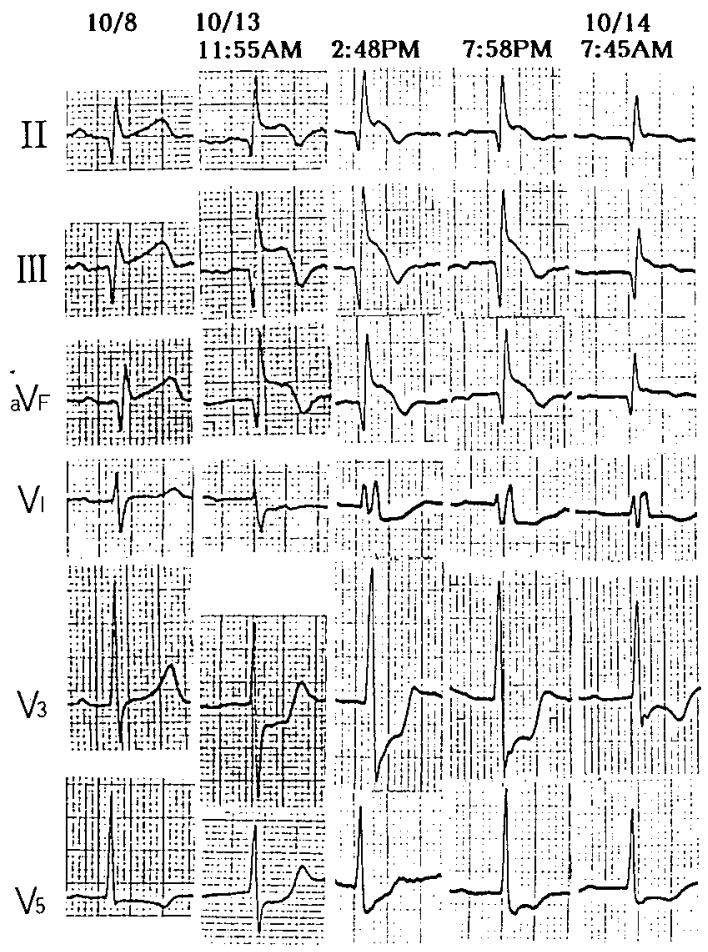

Fig 1. Electrocardiograms (ECGs) recorded serially after the onset of acute MI.

ECGs were recorded on the first hospital day (10/8), before $(10 / 13$ 11:55 am) and after admission to our hospital (10/13 $2: 48 \mathrm{pm}$ and $10 / 13$ 7:58 pm) on the sixth hospital day, and on the seventh hospital day (10/14). RBBB and first-degree $\mathrm{AV}$ block were present $6 \mathrm{~h}$ before the patient's death on $10 / 14$.
IVS.

The basal portion of the IVS, including the AV conduction system, was evaluated histologically using the serial sectioning method ${ }^{2,5}$ (Fig 3). Hemorrhagic necrosis was present in the prenodal atrial myocardium. The AV node and the penetrating portion of the His bundle were unaffected. The branching portion of the His bundle exhibited mild degeneration of the conducting cells adjacent to the area of myocardial necrosis. Part of the LBB could not be identified because of myocardial dissection, and part of it was necrotic. None of the RBB showed necrosis, and no MI was observed near the RBB. However, the proximal and distal parts of the second portion showed chronic damage (fibrous replacement) of the conducting cells, and the ratio of residual conducting cells to the total cross-sectional area of the RBB was 25 to $50 \%$.

\section{Discussion}

Some pathologic studies of the AV conduction system have been performed in patients with acute posterior MI. However, while patients with AV block were included in such studies, none of the patients had RBBB. Most of the studies indicated a poor correlation between AV block and the pathology of the lesion in the AV conduction system, as would be expected
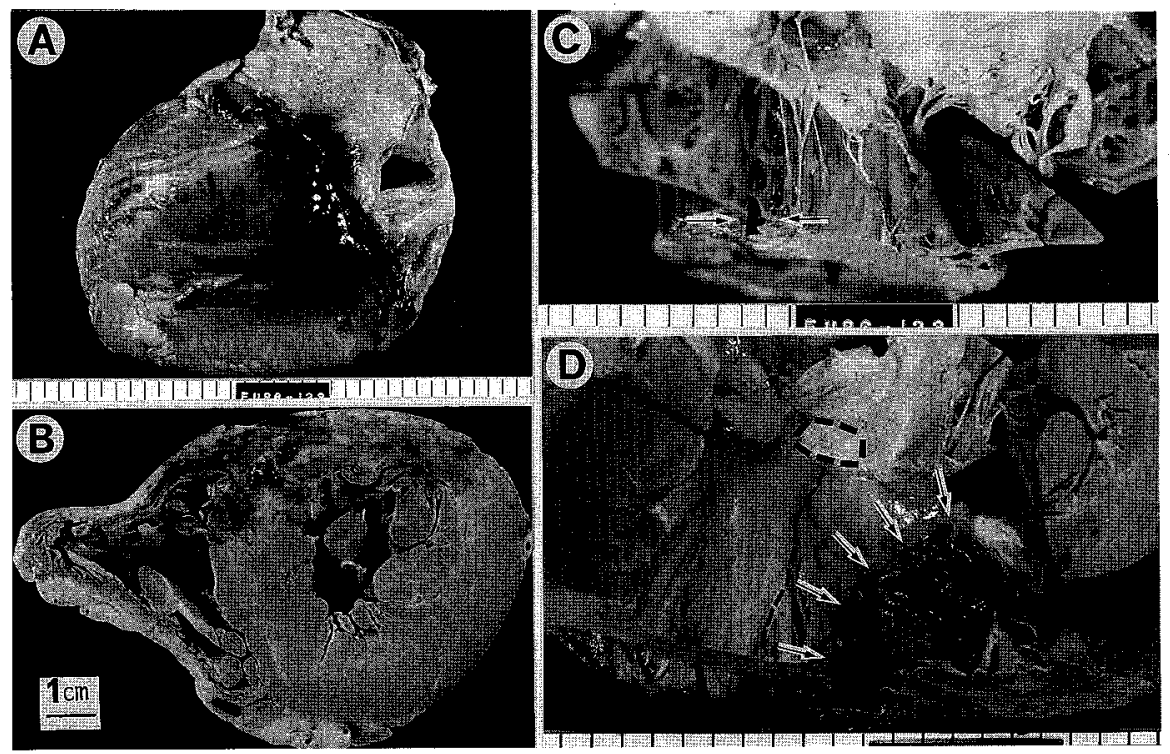

Fig 2. Pathology of the heart.

A: Posterior view of the heart. Subepicardial hemorrhage is apparent over the posterior walls of the left and right ventricles. B: Horizontal section of the ventricles. A recent MI with mural hemorrhage is observed. C: Internal view of the right ventricle. Arrows indicate a small perforation at the posterior edge of the IVS. D: Internal view of the left ventricle. A large myocardial dissection (arrows) extends to the membranous portion of the IVS, which is encircled by a thick broken line. The vertical broken line indicates the anterior border between the infarcted and noninfarcted portions of the IVS. 

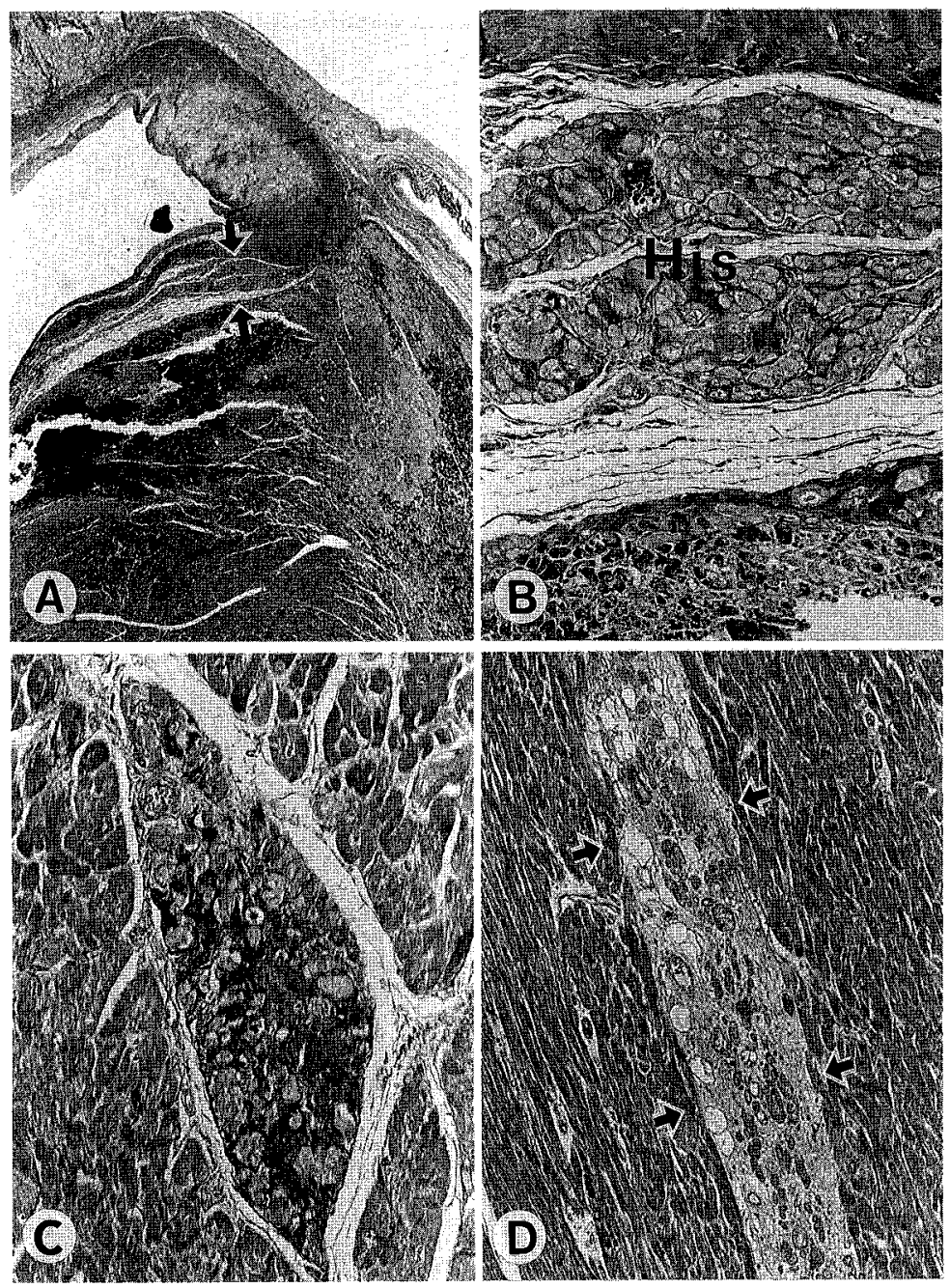

Fig 3. Histologic findings of the AV conduction system (Massson's trichrome stain). A: The branching portion of the His bundle (arrows) is adjacent to the.MI (darkly stained area). Original magnification $\times 13$. B: The conducting cells of the His bundle show mild degeneration. Infarcted myocardium with inflammatory infiltrates is seen at the bottom; the central fibrous body is at the top (original magnification $\times 130)$. C, D: Proximal (C) and distal (D) parts of the second portion of the RBBB show a moderate extent of fibrous replacement of the conducting cells (original magnification $\times 130$ and $\times 80$, respectively).

from the transient nature of AV block that develops in a patient with an acute posterior MI. Necrosis of the prenodal atrial myocardium as a pathologic change corresponding to clinical $\mathrm{AV}$ block was reported by Bilbao et al. Prenodal atrial necrosis in the present patient might have led to a prolongation of the $\mathrm{PQ}$ interval.

There are 2 pathologic studies of the AV conduction system ${ }^{7,8}$ in patients with a combination of a ruptured IVS and a posterior MI, including 5 cases of AV block? Three of these 5 had myocardial cavitation and tunneling dissection that penetrated upward into the IVS. The entire His bundle was protected from the dissecting MI by the mechanical strength of the central fibrous body. However, the AV node showed extensive necrosis in all 5 cases. The dissection could have penetrated a weak point of the posterior margin of the junction between the atrial and ventricular septa, and then proceeded anteriorly within the atrial septum toward the AV node. Rupture of the IVS in these 5 cases may have played a major role in the development of AV block?

Our patient presented with persistent RBBB: necropsy revealed a posterior MI with a ruptured IVS. The AV node and the proximal portion of the His bundle showed no ischemic involvement, as described previously? but the MI reached the distal portion of the His bundle and involved part of the LBB. This 
unusual extension of the MI is probably ascribed to myocardial dissection, but may be due to the discontinuation of collateral blood flow from the right coronary artery to the anterior IVS that had been perfused by the severely narrowed left anterior descending artery. Although the MI did not involve the RBB, chronic damage of the RBB appeared to be the pathologic basis of RBBB? Mechanical stretching of the RBB caused by the change in hemodynamics produced by a rupture of the IVS may also have played a role in the development of RBBB.

Based on the experience of our patient, a newly acquired RBBB during acute posterior MI is not likely due to ischemic damage to the RBB. Additional factors that may be involved in the development of RBBB should be considered, including those that influence the extension of MI, particularly in the anterior IVS, preexisting chronic damage to the RBB, and mechanical stress to the RBB caused by mechanical complications.

\section{References}

1. Lie KI, Wellens HL, Shuilenburg RM: Bundle branch block and acute myocardial infarction. In: Wellens HJ, Lie KI, Lanse MJ editors, The Conduction System of The Heart. Leiden, Stenfert Kroese, 1976; 663-672

2. Okabe M, Fukuda K, Nakashima Y, Hiroki T, Arakawa K, Kikuchi K: A quantitative histopathological study of right bundle branch block complicating acute anteroseptal myocardial infarction. Br Heart $J$ 1991; 65: 317-321

3. James TN, Burch GE: Blood supply of the human interventricular septum. Circulation 1958; 17: 391-396

4. Frink RJ, James TN: Normal blood supply to the His bundle and proximal bundle branches. Circulation 1973; 47: $8-18$

5. Fukuda K, Nakata Y, Okada R, Takagi T: Histopathological studies on the conduction system of complete right bundle branch block with special references to configuration of QRS complex. Jpn Heart J 1979; 20: 831-841

6. Bilbao FJ, Zabalza IE, Vilanova JR, Froufe J: Atrioventricular block in posterior acute myocardial infarction: a clinicopathologic correlation. Circulation 1987; 75: 733-736

7. James TN: De subitaneis mortibus. XXIV. Ruptured interventricular septum and heart block. Circulation 1977; 55: 934-936

8. Hutchins GM: Rupture of the interventricular septum complicating myocardial infarction: Pathological analysis of 10 patients with clinically diagnosed perforations. Am J Cardiol 1979; 97: 165-173 